\title{
ON THE LOSSES OF PROPELLING POWER IN THE PADDLE-WHEEL.
}

Bx R. H. Thurston, First Assistant Engineer, Assist. Prof. Nat. Phil., U.S. N.

(Member of the Institute.)

Is a marine zteam engine of good design and in good order, about seven per centum of the indicated horse-power may be expected to be consumed in the moving of the parts of the machinery itself, the remaining ninety-three por centum being applied to the propelling instrument.

Where the radial paddle-wheel is used, a considerable part of the work done by the wheel is expended uselessly in forcing the water downward, or in lifting it; and of that power which finally acts horizontally, a part is unavoidably lost in setting in motion the water upon which the wheel acts. Thus the power exerted by the engines is considerably greater than that exerted by the ship in resisting motion.

To ascertain the amount of these losses, when the vessel is at rest, as on the instant of starting the engines, the first of these losses, that from oblique action, is easily calculated thus:-

Measure the length of the immersed arc described by the centre of pressure of the floats, and the length of its chord. 'The ratio of the squares of these quantities will give the ratio of total power exerted to power expeuded horizontally by the wheel.

When the vessel moves ahead, the conditions of the problem are at once greatly changed.

The floats, then, instead of moving in a circle through the water, dezcribe the curve shown in Fig. 1.

They then move through the water obliquely, and with velocities varying every instant.

T'his forward movement gives, to some extent, a "feathering" action to the floats, and with a properly proportioned wheel, the loss from oblique action becomes very greatly dimirished.

The path of the float $\mathrm{R} c$ being in the direction $c d$ instead of the direction $a b$, the principal portion of the work is done in the lower portion of the path of the float, where there is less oblique action.

Nolicing the positions assumed by the floats at different points $c, s, l, \& c .$, and the direction of their mution, it becomes eviclent, also, that the amount of "dip" influences the arnount of loss from oblique action very greatly when the vessel moves abead, as well as in the previous case, and the mosteconomical wheel will be that in which the sum of the losses from dip anl slip are reduced to the greatest extent.

To asrertuin the amount of loss of power in oblique artion:-

See lige. 2.-Let $v=$ velocity of paddle-float at cents e of pressure.

$v=$ volocity of vessel.

$a=$ angle included between an arm $\mathrm{cA}$ and horizontal line through $c$.

$\beta=$ any other angle as $\mathrm{B} \mathrm{C} v$.

$\mu=\frac{v}{\mathbf{v}^{*}} \quad r=$ radius. 


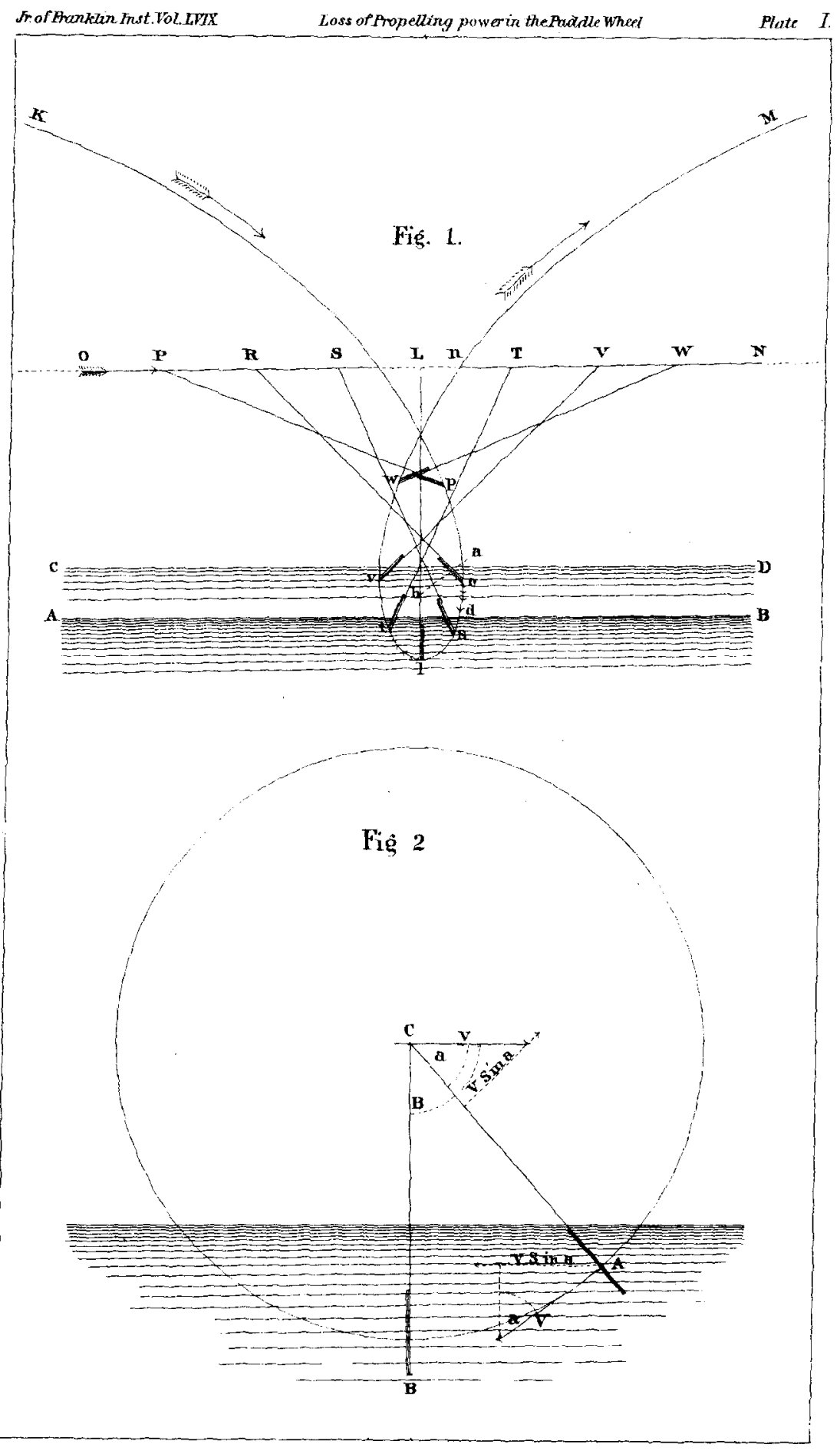


The tangential velocity of the centre of pressure of any float A c will be $(\mathrm{v}-v$ sin. $a)=\mathrm{v}(1-\mu \sin . a)$, and the horizontal velocity through the water will be ( $\mathrm{V} \sin . \alpha-v)=\mathrm{V}(\sin . \alpha-\mu)$.

The normal pressure on a unit of area of the float will be measured by the square of its velocity in the direction of that pressure, or $\mathrm{V}^{2}(1-\mu \sin a)^{2}$, and the total work done by the engine in turn: ing the float through any arc $\mathrm{A} B$, will be-

$$
r \mathrm{v}^{2} \int_{a}^{\beta}(1-\mu \sin . a)^{2} d a, \quad \text {. . . . . }
$$

The horizontal component of the pressure on the float, which only is useful in propelling the ship, will bc--

$$
\mathrm{v}^{2}(1-u \sin . a)^{2} \sin . a \text {. }
$$

While the float is moving through the arc $r d a$ in the time $d t=\frac{r d a}{\mathrm{v}}$, this horizontal resistance will be met through a space $v(\sin . a-\mu) d t=r d a(\sin . a--\mu)$, and the power expended horizontally upon the water while the wheel moves through any are A $\mathrm{B}$ will, therefore, be expressed by-

$$
r v^{2} \int_{a}^{\beta}(1-\mu \sin . \alpha)^{2} \sin . a(\sin , a-\mu) d a, \quad .
$$

But while this amount of work is expended upon the water, the wheel must, in consequence of the slip, do a greater amount of work in a proportion varying at each point with the slip, and the total horizontal work of the wheel will be obtained by multiplying the expression (2) by the factor $\frac{\mathrm{v}}{\mathrm{V}(1-\mu \sin . a)^{\circ}}$.

The total amount of work done in this direction, then, while passing through the are $\mathrm{A} \mathrm{B}$, will be, including slip,-

$$
r \mathrm{~V}^{2} \int_{a}^{\beta}(1-\mu \sin . \alpha)(\sin . a-\mu) \sin . a d a, \quad .
$$

The ratio of (3) to (1) will be the ratio of gross horizontal work to the total work done on the wheel.

Integrating between limits, this ratio becomes--

$\frac{(3 .)}{(1 .)}=\frac{\frac{\mu^{2}+1}{2}(\beta-a)+2 \mu(\cos \beta-\cos a)-\frac{\mu}{3}\left(\cos ^{3} \beta-\cos ^{3} a\right)-}{\frac{\mu^{2}+2}{2}(\beta-a)+2 \mu(\cos \beta-\cos \alpha)-\frac{\mu^{2}}{4}}$ $\frac{\frac{u^{2}+1}{4}(\sin .2 \beta-\sin .2 a)}{(\sin .2 \beta-\sin .2 a)}$

In this equation, making $a$ the angle of entrance of the meat centre of pressure of the float and calling $\beta=90^{\circ}, \frac{v}{\mathrm{v}}$ being known; 
the loss of power in any wheel by oblique action may be obtained by subtracting the ratio resulting from our final equation from unity.

T'he following are data assumed and results obtained as above:

\begin{tabular}{|c|c|c|}
\hline Second case. & Third case. & Fourth case. \\
\hline 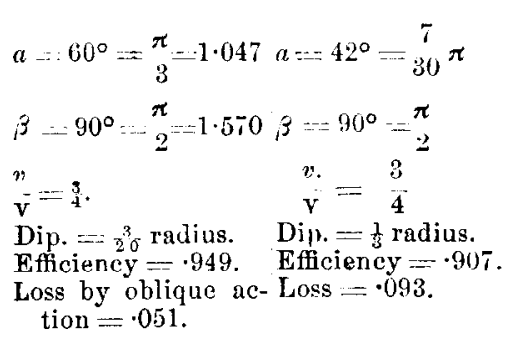 & $\begin{array}{l}a=30^{\circ}=\frac{\pi}{6} \\
\beta=90^{\circ}=\frac{\pi}{2} \\
v=\frac{3}{4} \\
\mathbf{v} \\
\text { Dip. }=\frac{1}{2} \text { radius. } \\
\text { Effic. }=83 . \\
\text { Loss }=\cdot 17 .\end{array}$ & $\begin{array}{l}\alpha=0^{\circ}=0 . \\
\beta=90^{\circ}=\frac{\pi}{2} \\
y=\frac{3}{4} \\
\vec{v}=- \\
\text { Dip. = to centre. } \\
\text { Effic. }=70 . \\
\text { Loss }=\cdot 30 .\end{array}$ \\
\hline
\end{tabular}

The first case corresponds to that of light river steamboats, the second to that of ocean steamers, while the third is an exaggerated, though not remarkably rare case. The fourth case is given simply in illustration of the use of the formula; it, of course, is unknown in practice.

Here the efficiency and loss are expressed, not in fractions of total indicated horse-power, but of the power applied to the wheel, and, referred to I. H.P., those losses would be $\cdot 047, \cdot 086, \cdot 158$ and $\cdot 28$, respectively.

If it is required to find the loss of power by oblique action and slip combined, and thus to ascertain if the designer of the hull has fulfilled his guarantees, the net power expended in simply overcoming the resistance of the ship must be obtained. The amount of horizontal expenditure of power just obtained must, in this case, be reduced by multiplying by the ratio $\frac{\mathrm{V}-\mathrm{v}}{\mathrm{V}}$, or it may be taken from (2).

In the examples given above, the slip is assumed to be known, and in each case is taken at 25 per cent., and this net power usefully expended is found, after making this reduction, to be $\cdot 714, \cdot 685$, $\cdot 631$ and 52 of total I. H. P.

In the fourth case, the slip would actually be very much greater than is assumed above, and the efficiency would probably fall below twenty per centum of the indicated horse-power.

Our results agree very closely with those deduced from experi. ment; and the method by which they are obtained has, we believe, never before been published.

U. S. Naral Academy, Annapolis, Md., Dec., 1866. 